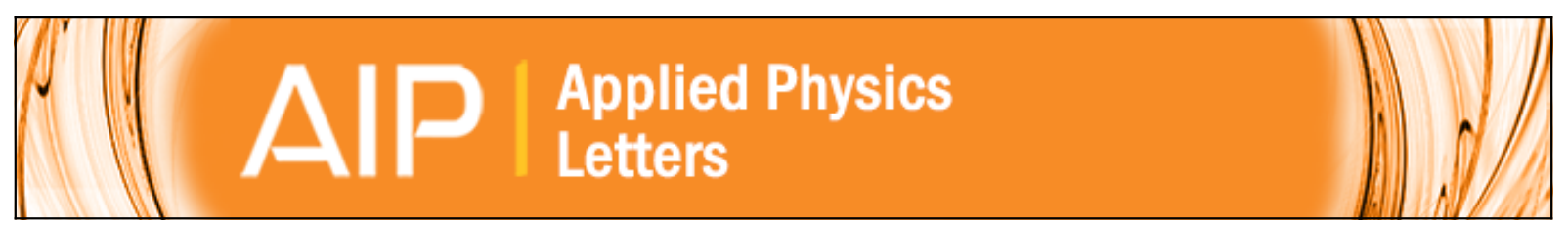

Highly efficient semitransparent tandem organic solar cells with complementary absorber materials

Jan Meiss, Torben Menke, Karl Leo, Christian Uhrich, Wolf-Michael Gnehr, Stefan Sonntag, Martin Pfeiffer, and Moritz Riede

Citation: Applied Physics Letters 99, 043301 (2011); doi: 10.1063/1.3610551

View online: http://dx.doi.org/10.1063/1.3610551

View Table of Contents: http://scitation.aip.org/content/aip/journal/apl/99/4?ver=pdfcov

Published by the AIP Publishing

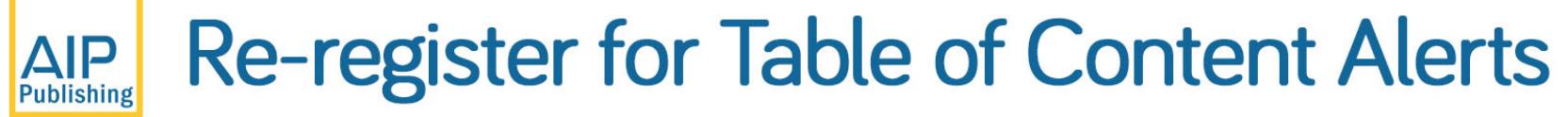

Create a profile. 


\title{
Highly efficient semitransparent tandem organic solar cells with complementary absorber materials
}

\author{
Jan Meiss, ${ }^{1}$ Torben Menke, ${ }^{1}$ Karl Leo, ${ }^{1}$ Christian Uhrich, ${ }^{2}$ Wolf-Michael Gnehr, ${ }^{2}$ \\ Stefan Sonntag, ${ }^{2}$ Martin Pfeiffer, ${ }^{2}$ and Moritz Riede ${ }^{1, a)}$ \\ ${ }^{1}$ Institut für Angewandte Photophysik, Technische Universität Dresden, 01062 Dresden, Germany \\ ${ }^{2}$ Heliatek GmbH, Treidlerstrasse 3, 01139 Dresden, Germany
}

(Received 4 May 2011; accepted 22 June 2011; published online 25 July 2011; corrected 14 December 2011)

We present highly efficient, semitransparent small molecule organic solar cells. The devices employ an indium tin oxide-free top contact, consisting of thin metal films. An additional organic layer is used to enhance light outcoupling. The solar cell incorporates two stacked subcells, each containing a donor:acceptor bulk heterojunction. The two subcells have complementary absorbers, with separate blue $\left(\mathrm{C}_{60}\right)$, red (fluorinated zinc phthalocyanine), and green (dicyanovinyl oligothiophene derivative) absorbing molecules. A power conversion efficiency of $4.9 \pm 0.2 \%$ is obtained for the device having an average transmission of $24 \%$ in the visible range. (C) 2011 American Institute of Physics. [doi:10.1063/1.3610551]

Organic solar cells are advancing towards commercial maturity, and new materials and concepts have resulted in certified efficiencies of $8.3 \%$ for small molecule organic solar cells (SMOSC) and for polymer devices. ${ }^{1}$ Organic solar cells are currently particularly interesting for niche markets where cost-efficient, lightweight, and flexible solutions are advantageous, e.g., small consumer electronics or smallscale current generation in remote areas.

However, building-integrated photovoltaics (BIPV) will also play an important role in the future. ${ }^{2}$ One field of BIPV includes the possibility of electricity-generating windows by fabricating semitransparent organic solar cells, which would additionally provide sun shading. After initial research lead to power conversion efficiencies $\eta$ well below $1 \%$ for semitransparent SMOSC, ${ }^{3,4}$ the development of optimized semitransparent metal contacts ${ }^{5}$ and light incoupling layers ${ }^{6,7}$ finally lead to $\eta>2 \%$ in zinc phthalocyanine $\mathrm{ZnPc}: \mathrm{C}_{60^{-}}$ based devices. $^{8}$

We present an optimized transparent tandem SMOSC that employs, in addition to the acceptor $\mathrm{C}_{60}$, two recently developed donor materials, the green absorber $\alpha, \omega$-bis(dicyanovinyl-sexithiophene)-Bu(1,2,5,6) (DCV6T) and the red absorber fluorinated $\mathrm{ZnPc}\left(\mathrm{F}_{4}-\mathrm{ZnPc}\right)$. The structures of all absorber materials are shown in Fig. 1(a). The devices with the stack shown in Fig. 1(b) exhibit high $\eta^{*}=4.9 \%$ while still being transparent, as illustrated in the module photo in Fig. 1(c).

The solar cells are fabricated as described elsewhere. ${ }^{8}$ The devices employ the pin-concept where highly conductive, doped charge transport layers sandwich the intrinsic absorber zone. An electron transport layer (ETL) of $n-C_{60}$ is used for ohmic contact between indium tin oxide (ITO) and organic layers. The first heterojunction $\left(\mathrm{F}_{4}-\mathrm{ZnPc}: \mathrm{C}_{60}\right)$ is embedded between an intrinsic ETL and a hole transport layer (HTL). A thick (135 nm) p-doped layer is used as optical spacer to optimize the interference pattern within the device. A highly doped, organic pn-contact is used as recombination

${ }^{\text {a)} E l e c t r o n i c ~ m a i l: ~ m o r i t z . r i e d e @ i a p p . d e . ~}$ contact (RC) of the two subcells, which are connected in series. ${ }^{9}$ The topmost absorber blend (DCV6T: $\mathrm{C}_{60}$ ) covers the green and blue parts of the spectrum. A further HTL and a second RC lead to efficient transport towards the semitransparent top electrode. A combination of $1 \mathrm{~nm} \mathrm{Al}$ with $21 \mathrm{~nm}$ $\mathrm{Ag}$ is used as a transparent top contact. On top of the metal electrode, a $90 \mathrm{~nm}$ thick capping layer of tris(8-hydroxyquinolinato)-aluminum $\left(\mathrm{Alq}_{3}\right)$ is used for increased light outcoupling ${ }^{10}$ out of the solar cell.

DiNPB is purchased from Sensient AG (Germany). DCV6T is provided by Heliatek $\mathrm{GmbH}$ (Germany), $\mathrm{F}_{4}-\mathrm{ZnPc}$ by BASF SE (Germany), and $\mathrm{C}_{60}$ by BuckyUSA (USA). The dopants NDP9 and NDN1 are purchased from Novaled AG (Germany). All organic materials except the dopants had been purified at least twice by vacuum gradient sublimation. Instead of the commonly used tetra-fluoro-tetracyano-quinodimethane $\left(\mathrm{F}_{4}\right.$-TCNQ) or $\mathrm{WO}_{3}$ to p-dope materials with high ionization potentials and acridine orange base as n-dopant, NDP9 and NDN1 are used because of a better processability.

Typical solar cell areas are approximately $6.26 \mathrm{~mm}^{2}$. Further storage and characterization are performed under ambient conditions. The standard $J(V)$ measurements are carried out using simulated AG 1.5G sun light from a SC1200 sun simulator (K.H. Steuernagel, Germany). The intensity is monitored by a Si diode that is calibrated using a reference diode from the Fraunhofer ISE CalLab, Germany; the values are not corrected for spectral mismatch.

The external quantum efficiency (EQE) is measured as described elsewhere. ${ }^{8}$ Additional constant bias illumination is provided by LEDs: orange-red and blue LEDs with an emission peak at $640 \mathrm{~nm}(20 \mathrm{~nm}$ spectral half-width) and $470 \mathrm{~nm}$ (25 nm spectral half-width), respectively, are used. The approximate intensity of the light bias is $5 \mathrm{~mW} / \mathrm{cm}^{2}$.

The solar cell $J(V)$ curves under illumination and in the dark are shown in Fig. 2. The devices exhibit high fill factor (FF) of $61 \%$ and an open circuit voltage of $1.54 \mathrm{~V}$. The open circuit voltage originates from the sum of the $\mathrm{F}_{4}-\mathrm{ZnPc}(0.7$ $\mathrm{V})$ and DCV6T $(0.88 \mathrm{~V})$ subcells. ${ }^{11}$ The measured value of this tandem device very closely corresponds to the sum of 


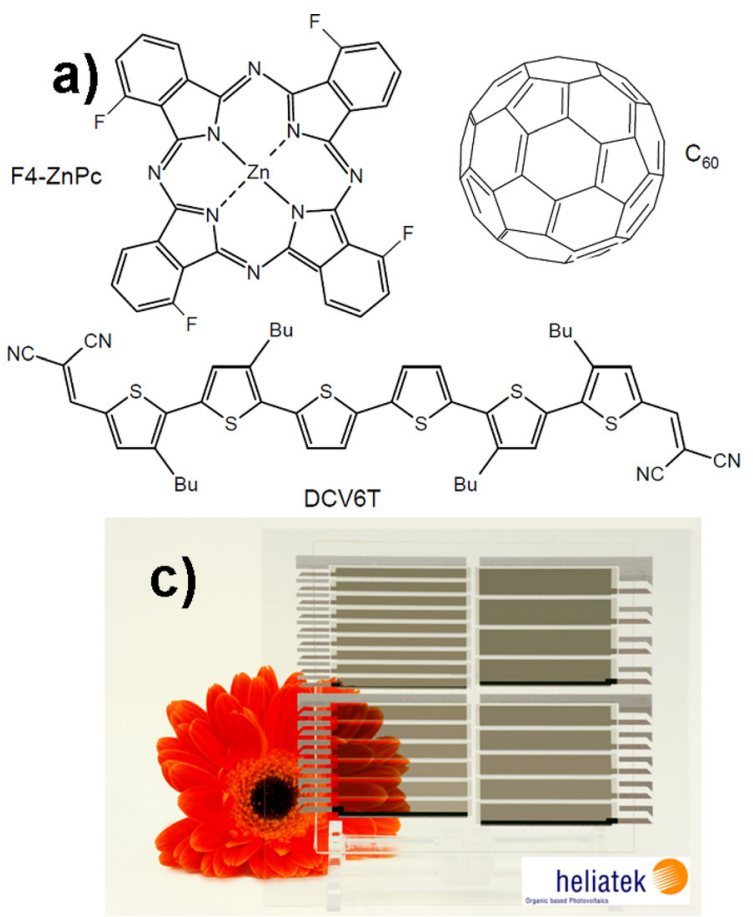

both subcells; combined with the high FF, this hints at a very efficient recombination contact between both subcells. ${ }^{9}$

The short circuit current density at $100 \mathrm{~mW} / \mathrm{cm}^{2}$ simulated sunlight is measured to be $J_{\mathrm{SC}}^{*}=(5.2 \pm 0.1) \mathrm{mA} / \mathrm{cm}^{2}$, which leads to a power conversion efficiency of $\eta^{*}=4.9 \pm 0.2 \%$. While this value competes with some of the best regular small molecule tandem devices seen in the literature, $^{12-14}$ the SMOSC in the current work additionally exhibits transparency in the visible range.

Reflectance $R$ and transmittance $T$ measurements are performed on a Lambda 900 UV/VIS/NIR spectrometer (Perkin Elmer); the corresponding data are shown in Fig. 3. An average $T=24 \%$ is observed in the visible range (400$800 \mathrm{~nm})$.

The data show that there is a fundamental difference in reflectance, depending on whether one measures from the bottom (through the glass substrate and ITO) or from the top (through light incoupling layer and metal contact). The most decisive factor concerning the optical effects, and an impor-

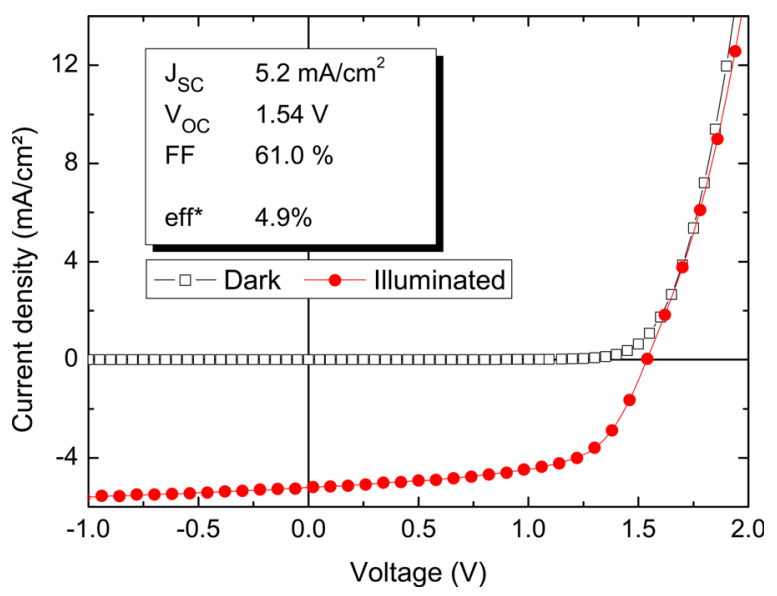

FIG. 2. (Color online) $J(V)$ measurements of the semitransparent SMOSC. Squares: dark; circles: illuminated through ITO at $100 \mathrm{~mW} / \mathrm{cm}^{2}$. tant influence on the photocurrent, is interference at the ultra-thin metal top contact. If light is illuminated through the bottom, i.e., the substrate and ITO, a significant part of what is not absorbed in the first pass is reflected at the metal top contact. This results in a second pass through the absorber layers and leads to a high photocurrent due to the microcavity effect.

In contrast to that, if the device is illuminated from the top, reflection of the incoming wave is strongly increased; furthermore, the light that is transmitted through the top contact makes only one pass through the absorber layers before being outcoupled through ITO and glass, such that the device performance is significantly lower. The transmittance is independent of the illumination orientation, but absorption and reflection are strongly influenced.

To verify that both subcells contribute to the tandem devices, EQE measurements are performed with different kinds of bias illumination: green bias light to flood the

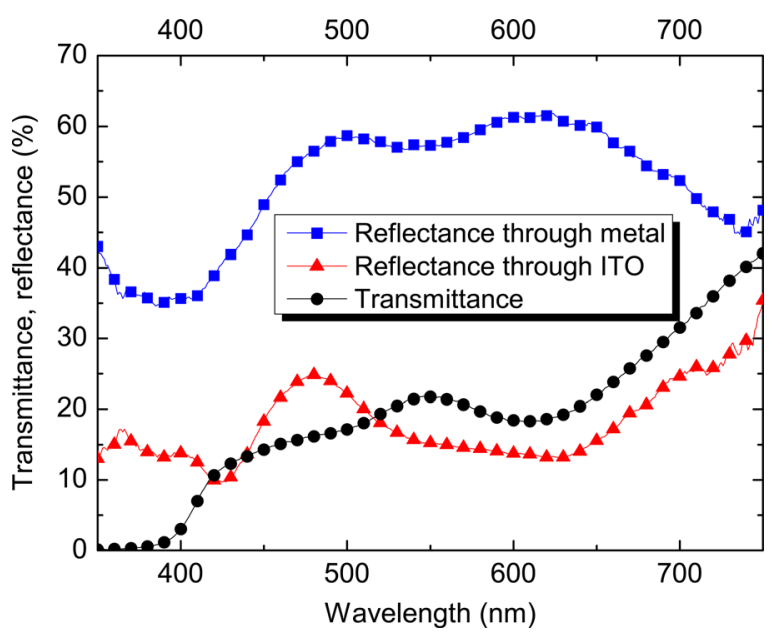

FIG. 3. (Color online) Reflectance, measured through ITO (triangles) or through the metal contact (squares), and transmittance (circles). 
DCV6T-containing subcell and characterize the $\mathrm{F}_{4}-\mathrm{ZnPc}$ containing subcell and red bias light to study the green subcell. The resulting data are depicted in Fig. 4 (top). The peak EQE are observed at $550 \mathrm{~nm}$ with $32 \%$ for DCV6T and at $640 \mathrm{~nm}$ with $31 \%$ for $\mathrm{F}_{4}-\mathrm{ZnPc}$. Altogether, we observe coverage of a broad range of the solar spectrum from 450 to 750 $\mathrm{nm}$. EQE at $<450 \mathrm{~nm}$ results mostly from the fullerene in the blend layers; however, at $\lambda<400 \mathrm{~nm}$, the solar spectrum has only a relatively small photon flux, such that this wavelength range only contributes weakly to the total device photocurrent.

Calculations using a numerical algorithm based on the transfer matrix approach and Poynting vector computations ${ }^{15}$ indicate that the photocurrent of the green subcell $J_{\text {green }}$ is in all cases smaller than $J_{r e d}$ of the $\mathrm{F}_{4}-\mathrm{ZnPc}$ subcell and is hence current-limiting. $J_{\text {green }}$ for a wide thickness variation of semitransparent $\mathrm{Ag}$ top contacts and $\mathrm{Alq}_{3}$ capping layers is shown in Fig. 4 (bottom). The device presented in this letter $\left(21 \mathrm{~nm} \mathrm{Ag}\right.$ and $\left.90 \mathrm{~nm} \mathrm{Alq}{ }_{3}\right)$ is marked with a black square.

Generally, thicker metal layers lead to higher photocurrents. The difference can be as large as $21 \%$, comparing 15 $\mathrm{nm}$ and $25 \mathrm{~nm} \mathrm{Ag}$ at $50 \mathrm{~nm}$ capping layer thickness, but can
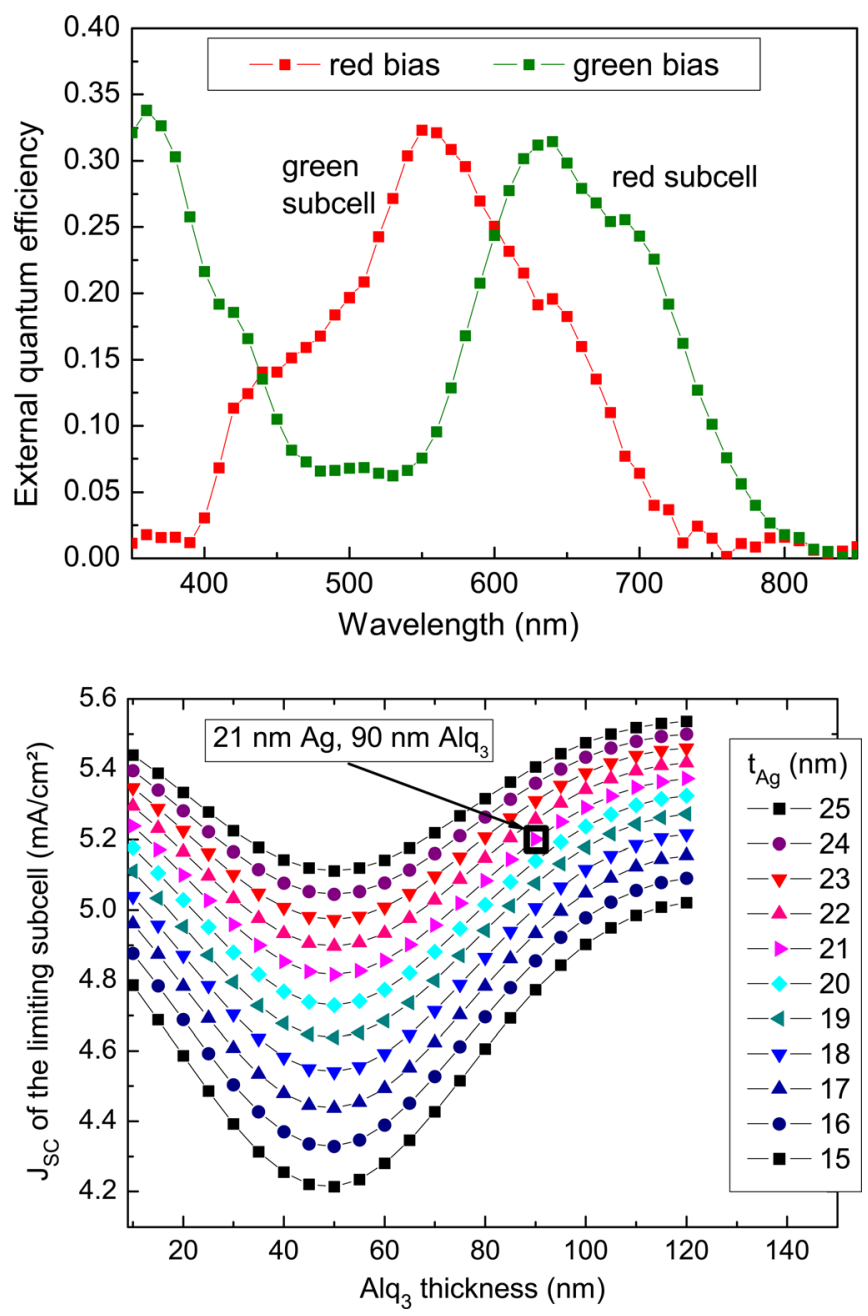

FIG. 4. (Color online) Top: EQE measurements under red bias (to probe the green-absorbing DCV6T: $\mathrm{C}_{60}$ subcell) and green bias (to characterize the $\mathrm{F}_{4}$ $\mathrm{ZnPc}: \mathrm{C}_{60}$ subcell). Bottom: calculated photocurrent of the limiting, greenabsorbing subcell for different layer thicknesses of $\mathrm{Alq}_{3}$ and $\mathrm{Ag}$. also be smaller ( $10 \%$ at $\left.120 \mathrm{~nm} \mathrm{Alq}_{3}\right)$. This is explained by the stronger reflection at the metal back electrode if the device is illuminated through the ITO bottom contact: the constructive interference in the absorber layers is enhanced, improving photon absorption. However, at the same time, the transmission is decreased, such that a compromise must be found for optimized semitransparent applications.

The $\mathrm{Alq}_{3}$ capping layer is a second means to controlling interference and hence optical properties and device performance. ${ }^{6,7}$ The data demonstrate that at any given fixed metal thickness, the capping layer, even though it is outside the active layers, strongly influences the photocurrent by up to $20 \%$.

The $J_{S C}^{*}$ which we observe in our experiments corresponds, within the margin of error, closely to the simulated value for the current-limiting green subcell. It must be considered that simulations rely on experimental input (e.g., density, layer thickness and homogeneity, and optical constants) and may thus deviate slightly from the measurements. However, this good agreement suggests that the SMOSC utilizes absorbed photons very efficiently, and recombination losses in the green subcell are small.

In conclusion, we present highly efficient semitransparent SMOSC, demonstrating that a good compromise of transparency $(24 \%)$ and efficiency $(4.9 \pm 0.2 \%)$ is possible. This is achieved using optimized multi-layer metal top electrodes and donor materials to achieve high internal quantum efficiency. Further tests (not shown here) also revealed that the device stack can also be transferred to module-relevant areas of up to $400 \mathrm{~mm}^{2}$.

The current work is supported by the German Federal Ministry of Education and Research (BMBF) in the framework of the InnoProfile (03IP602) and OPEG (13N9716) projects. We thank Dr. P. Erk, Dr. J. Hwang, and Dr. S. Sundarraj (BASF SE) for providing $\mathrm{F}_{4}-\mathrm{ZnPc}$.

${ }^{1} 8.3 \%$ efficient SMOSC on $1.1 \mathrm{~cm}^{2}$ area (Heliatek/IAPP); $8.3 \%$ efficient polymer device (Konarka).

${ }^{2}$ A. Henemann, Renewable Energy Focus 9(1), 14 (2008).

${ }^{3}$ R. Bailey-Salzman, B. P. Rand, and S. R. Forrest, Appl. Phys. Lett. 88, 233502 (2006).

${ }^{4}$ R. Koeppe, D. Hoeglinger, P. A. Troshin, R. N. Lyubovskaya, V. F. Razumov, and N. S. Sariciftci, ChemSusChem 2, 309 (2009).

${ }^{5}$ J. Meiss, M. K. Riede, and K. Leo, J. Appl. Phys. 105, 063108 (2009).

${ }^{6}$ B. O'Connor, K. H. An, K. P. Pipe, Y. Zhao, and M. Shtein, Appl. Phys. Lett. 89, 233502 (2006).

${ }^{7}$ J. Meiss, N. Allinger, M. K. Riede, and K. Leo, Appl. Phys. Lett. 93, 103311 (2009).

${ }^{8}$ J. Meiss, K. Leo, M. K. Riede, C. Uhrich, W.-M. Gnehr, S. Sonntag, and M. Pfeiffer, Appl. Phys. Lett. 95, 213306 (2009).

${ }^{9}$ R. Timmreck, S. Olthof, K. Leo, and M. Riede, J. Appl. Phys. 108, 033108 (2010).

${ }^{10}$ Q. Huang, K. Walzer, M. Pfeiffer, V. Lyssenko, G. He, and K. Leo, Appl. Phys. Lett. 88(11), 113515 (2006).

${ }^{11}$ M. Riede, C. Uhrich, J. Widmer, R. Timmreck, D. Wynands, G. Schwartz, W.-M. Gnehr, D. Hildebrandt, A. Weiss, J. Hwang, S. Sudharka, P. Erk, M. Pfeiffer, and K. Leo, "Efficient Organic Tandem Solar Cells based on Small Molecules" (in press).

${ }^{12}$ D. Cheyns, B. P. Rand, and P. Heremans, Appl. Phys. Lett. 97, 033301 (2010).

${ }^{13}$ J. Drechsel, B. Maennig, F. Kozlowski, M. Pfeiffer, K. Leo, and H. Hoppe, Appl. Phys. Lett. 86, 244102 (2005).

${ }^{14}$ G. Dennler, H.-J. Prall, R. Koeppe, M. Egginger, R. Autengruber, and N. S. Sariciftci, Appl. Phys. Lett. 89, 073502 (2006).

${ }^{15}$ E. Centurioni, Appl. Opt. 44(35), 7532 (2005). 